\title{
Angiomyolipoma of the rib: A rare case report
}

\author{
GUANG-XIANG CHEN ${ }^{1}$, MAO-HUA WANG ${ }^{2}$, CHANG-LIE HU $^{3}$, GUANG-CAI TANG ${ }^{1}$ and FU-GANG HAN ${ }^{1}$ \\ Departments of ${ }^{1}$ Radiology and ${ }^{2}$ Anesthesiology, The Affiliated Hospital of Luzhou Medical College, Luzhou, \\ Sichuan 646000; ${ }^{3}$ Department of Radiology, Mianzhu Renai Hospital, Deyang, Sichuan 618200, P.R. China
}

Received May 28, 2015; Accepted October 16, 2015

DOI: $10.3892 / \mathrm{mco} .2015 .669$

\begin{abstract}
Angiomyolipoma is a benign mesenchymal tumor, most commonly arising from the kidney. With the exception of the liver, extrarenal angiomyolipoma is an infrequent entity that may be misdiagnosed as other tumors. Angiomyolipoma occurring in the skeleton is an extremely rare occurrence and often difficult to differentiate from other bone diseases. We herein present a case of angiomyolipoma in the rib of a 44-year-old male patient, a highly unusual location for this type of tumor. Angiomyolipoma of the skeleton usually exhibits an infiltrative tendency. Although this clinical entity is rare, angiomyolipoma should be considered in the differential diagnosis of skeletal lesions, particularly in patients presenting with imaging findings of fat within the lesions.
\end{abstract}

\section{Introduction}

Angiomyolipoma is a benign, mesenchymal hamartomatous neoplasm composed of variable combinations of adipose tissue, smooth muscle cells and abnormal blood vessels. Angiomyolipoma often arises from the kidney, but has also been described in extrarenal locations, such as the liver (1), uterus (2), and other infrequent sites (3-5). Angiomyolipomas arising from the skeleton are extremely rare and have been previously reported in the thoracic (6) and cervical spine (7), as well as the tibia (8). However, the radiological characteristics of angiomyolipomas arising from the skeleton have not been adequately described due to our limited experience with this type of tumor. Thus, the skeletal occurrence of angiomyolipoma represents a diagnostic challenge prior to surgery, in terms of unfamiliarity, unusual presentation and absence of characteristic imaging findings. We herein report a case of angiomyolipoma of the rib in a 44-year-old male patient that was diagnosed by pathological examination following surgical resection, and discuss the imaging findings of angiomyolipomas arising from the skeleton in combination with relevant published studies.

Correspondence to: Professor Guang-Xiang Chen, Department of Radiology, The Affiliated Hospital of Luzhou Medical College, 25 Taiping Street, Jiangyang, Luzhou, Sichuan 646000, P.R. China E-mail: cgx231y2002@163.com

Key words: angiomyolipoma, rib, computed tomography, skeleton

\section{Case report}

Patient history. A 44-year-old male patient presented $\sim 2$ months prior with a 3-year history of repeated attacks of right chest pain and was found to have a local mass in the right anterolateral chest. On physical examination, the mass was ovoid, firm, smooth, poorly mobile and tender to palpation. No pigmented lesions were observed in the skin. All the laboratory test results were within the normal range. Written informed consent was obtained from the patient for the publication of his medical details.

Examination. A chest computed tomography (CT) scan revealed that the mass was located in the right 8 th anterior rib, with localized expansive destruction and a discontinuous bone cortex. The central bone texture was irregularly destroyed and replaced with heterogeneous soft tissue density. The margin of the area of bone destruction was not very sharp, and there was some residual coarse and thickened bone cortex. The adjacent soft tissue was invaded by the lesion breaking through the cortex, and an oval soft tissue mass surrounding the area of bone destruction was observed. Maximum intensity projection and 3D volume-rendering revealed the enlargement of the right 8th anterior rib, with patchy destruction of the bone and internal septum (Fig. 1). The patient also underwent abdominal ultrasonography and a cerebral CT scan, which did not reveal any other lesions.

Treatment. The patient underwent surgical excision of the lesion with partial costectomy. Macroscopically, there was a $7.2 \times 5.5 \times 3.5-\mathrm{cm}$ well-circumscribed, reddish in color, firm mass in the proximal segment of the right 8 th rib adjacent to the costal cartilage. The right 8 th subcostal nerve and blood vessel were compressed by the tumor. On pathological examination, the tumor was confirmed to be an angiomyolipoma, consisting of mature adipocytes, spindle cells and vascular channels of various sizes (Fig. 2). The patient is being followed up by clinical physical examinations and CT scan and remains recurrence-free at 18 months postoperatively.

\section{Discussion}

Angiomyolipoma belongs to a family of tumors arising from perivascular epithelioid cells, referred to as PEComas, with the kidney being the most frequent site involved (9). Approximately $20 \%$ of renal angiomyolipomas are associated 

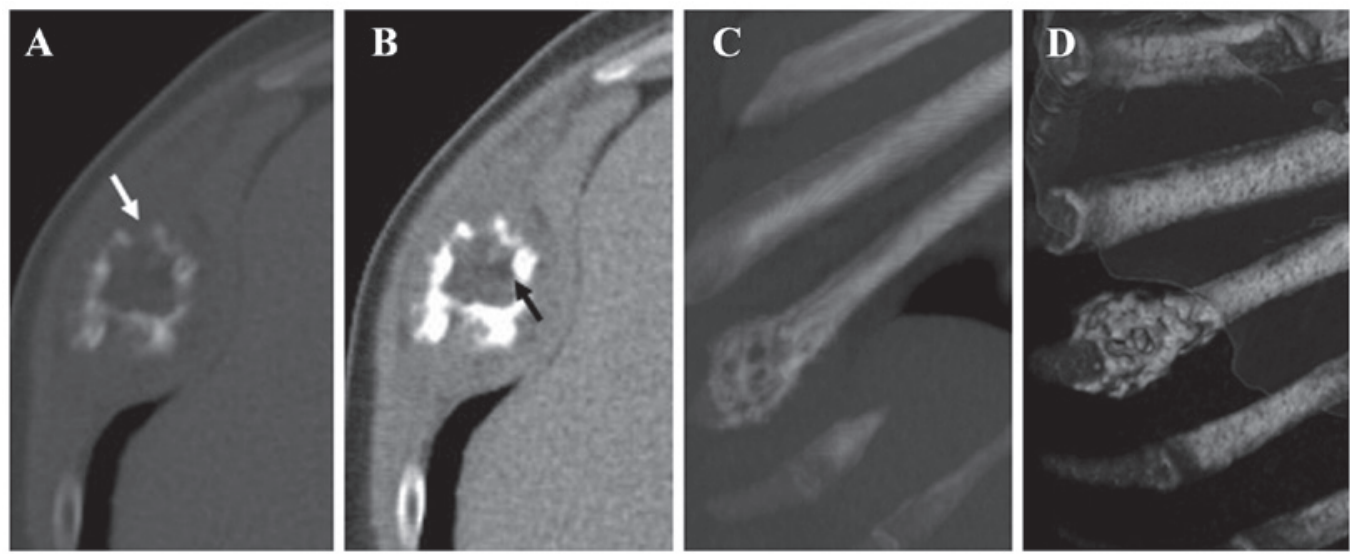

Figure 1. Computed tomography (CT) scan revealing an expansive destructive lesion in the right 8th anterior rib. (A) CT scan at bone window settings revealed an expansive destructive lesion and discontinuous bone cortex (arrow). (B) An oval soft tissue mass surrounding the bone destruction was observed at soft tissue window settings. There was a small amount of fat tissue within the lesion (arrow). (C and D) Maximum intensity projection and 3D volume-rendering revealed an enlargement of the right 8th anterior rib, within which patchy bone destruction and an internal septum were observed.
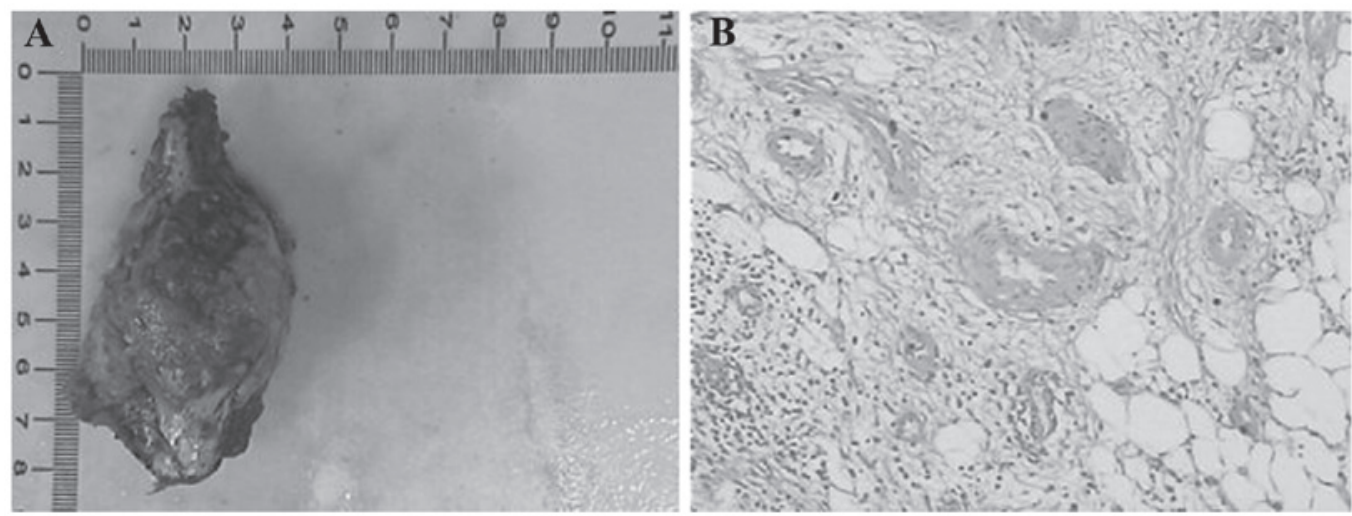

Figure 2. Macroscopic and histopathological characteristics of angiomyolipoma. (A) On gross examination, the angiomyolipoma was an oval, intact, reddish mass. (B) The microscopic examination of the resected tumor revealed that it consisted of mature adipocytes, spindle cells and blood vessels (hematoxylin and eosin staining; magnification, $\mathrm{x} 400$ ).

with tuberous sclerosis complex, which is a multisystemic disease with an autosomal dominant inheritance $(3,10)$. Extrarenal angiomyolipomas are rare, apart from those occurring in the liver, and occurrence in the skeleton is extremely rare. Owing to the lack of experience with this type of tumor and limited data in the literature reagrding the radiological manifestations of skeletal angiomyolipomas, this tumor may be easily misdiagnosed.

The preoperative diagnosis of angiomyolipoma may be difficult due to the variations in the ratio of the three components (fat, blood vessels and smooth muscle bundles) among different tumors, particularly tumors with a minimal amount of adipose tissue. Therefore, ascertaining the presence of fat is vital for the diagnosis of angiomyolipoma. CT and magnetic resonance imaging (MRI) are sensitive methods for detecting adipose tissue. The fatty component of angiomyolipoma displays low attenuation on CT and high signal intensity on T1- and T2-weighted MR images. In the present case, there was a small amount of fat tissue within the lesion, with a CT value of -38.4 Hounsfield units. Two previous cases of skeletal angiomyolipomas located in the thoracic and the cervical spine have been reported (6,7). Insabato et al (8) reported a case of primary monotypic epithelioid angiomyolipoma, a distinct and definable variant of angiomyolipoma, located in the tibia. We took notice of certain interesting findings in these previously reported cases of skeletal angiomyolipoma, as well as in the present case. All the patients were middle-aged or elderly men, and no case was associated with tuberous sclerosis complex. This is consistent with the majority of extrarenal angiomyolipoma cases, namely that extrarenal angiomyolipomas present mostly without evidence of tuberous sclerosis complex (2). The tumor morphology was usually consistent, with the tumors being round or oval in shape. Spinal angiomyolipomas were relatively common, accounting for two cases in these patients. The skeletal changes were mainly osteolytic or expansive destruction, mostly associated with cortical interruption. The margin of bone destruction was well-defined or unclear; a soft mass with a well-defined margin was occasionally found to surround the area of bone destruction. The two cases of spinal angiomyolipoma exhibited infiltrative properties and they were non-encapsulated, involving adjacent structures. In the present case, the CT scan revealed an expansive lesion with a surrounding well-defined soft mass. The findings were consistent with those of cases previously reported in the literature, in that the skeletal angiomyolipoma may break through the bone cortex and invade adjacent soft tissues. 
The radiological differential diagnosis for angiomyolipoma of the skeleton includes chondroma, osteofibrous dysplasia and tuberculosis (11-13). Chondroma is associated with characteristic calcifications within the lesion. Osteofibrous dysplasia presents with expansive destruction, with a thinner but continuous bone cortex and no soft mass surrounding the lesion. Skeletal tuberculosis may be differentiated through the characteristic sequestrum or calcification, as well as the soft tissue tuberculosis abscess surrounding the bone destruction. Infiltrative angiomyolipoma must be distinguished from various malignant tumors, such as sarcoma and metastatic tumors. In addition to identifying the presence of fat within the lesion, enhanced performance on CT and MRI may also contribute to the differential diagnosis of angiomyolipoma. The blood vessels in the tumor are strongly enhanced during the early phase of enhancement.

To the best of our knowledge, this is the first reported case of angiomyolipoma of the rib. Although angiomyolipoma is a tumor with a benign clinical course, angiomyolipomas of the skeleton usually exhibit infiltrative properties. When tumors of the skeleton in adult male patients display areas of fat within the lesion on imaging, angiomyolipoma of the skeleton should be considered, regardless of whether there is a well-defined soft mass surrounding the area of bone destruction.

\section{References}

1. Petrolla AA and Xin W: Hepatic angiomyolipoma. Arch Pathol Lab Med 132: 1679-1682, 2008.
2. Yaegashi H, Moriya T, Soeda S, Yonemoto Y, Nagura H and Sasano H: Uterine angiomyolipoma: Case report and review of the literature. Pathol Int 51: 896-901, 2001.

3. Oishi K, Fukuda S, Sakimoto H, Eto T, Takahashi M and Nishida T: Angiomyolipoma of the colon: Report of a case. Surg Today 39: 998-1001, 2009.

4. Saito M, Yuasa T, Nanjo H, Tsuchiya N, Satoh S and Habuchi T: A case of testicular angiomyolipoma. Int J Urol 15: 185-187, 2008.

5. Mikoshiba Y, Murata H, Ashida A, Saito N, Koga H, Uhara H and Okuyama R: Case of a cutaneous angiomyolipoma in the ear. J Dermatol 39: 808-809, 2012.

6. Sakaida H, Waga S, Kojima T, Kubo Y, Matsubara T and Yamamoto J: Thoracic spinal angiomyolipoma with extracanal extension to the thoracic cavity. A case report. Spine (Phila Pa 1976) 23: 391-394, 1998.

7. Jia N, Wang C, Liu HM, Yu H and Wang J: Angiomyolipoma occurred at the cervical spine. Joint Bone Spine 75: 620-621, 2008.

8. Insabato L, De Rosa G, Terracciano LM, Fazioli F, Di Santo F and Rosai J: Primary monotypic epithelioid angiomyolipoma of bone. Histopathology 40: 286-290, 2002.

9. Alvarez Alvarez C, Fernández Sanromán J, Fernández Castilla M and Antón Badiola I: Sporadic oral angiomyolipoma. Case report. Med Oral Patol Oral Cir Bucal 12: E391-E393, 2007.

10. Nelson CP and Sanda MG: Contemporary diagnosis and management of renal angiomyolipoma. J Urol 168: 1315-1325, 2002.

11. Qasem SA and DeYoung BR: Cartilage-forming tumors. Semin Diagn Pathol 31: 10-20, 2014.

12. Bethapudi S, Ritchie DA, Macduff E and Straiton J: Imaging in osteofibrous dysplasia, osteofibrous dysplasia-like adamantinoma, and classic adamantinoma. Clin Radiol 69: 200-208, 2014.

13. Hu N, Tan Y, Cheng Z, Hao Z and Wang Y; Hu N1: FDG PET/CT in Monitoring Antituberculosis Therapy in Patient With Widespread Skeletal Tuberculosis. Clin Nucl Med 40: 919-921, 2015. 